International Journal of Pure and Applied Mathematics

Volume 105 No. 4 2015, 751-761

ISSN: 1311-8080 (printed version); ISSN: 1314-3395 (on-line version)

url: http://www.ijpam.eu

doi: http://dx.doi.org/10.12732/ijpam.v105i4.15

\title{
ON GRAPH DOMINATION NUMBERS
}

\author{
V. Yegnanarayanan ${ }^{1} \S$, B. Logeshwary ${ }^{2}$ \\ ${ }^{1}$ Department of Humanities and Sciences \\ Sree Sastha Institute of Engineering and Technology \\ Chennai, 600123, INDIA \\ ${ }^{2}$ Research and Development Center \\ Bharathiar University \\ Coimbatore, 641046, INDIA
}

\begin{abstract}
In this paper, we determine for a simple graph $G$ on $n$ vertices and $m$ edges a variety of domination parameters such as connected domination number, outer connected domination number, doubly connected domination number, global domination number, total global connected domination number, 2-connected domination number, strong domination number, fair domination number, independence domination number etc.
\end{abstract}

Key Words: graph, domination number, connected domination number

\section{Introduction}

In this section, we introduce the notion and notation used in this paper.

For a graph $G=(V, E)$, let $|V(G)|=n$ and $|E(G)|=m$. The open neighborhood of a vertex $u \in V(G)$, denoted as $N(u)$, consists of all vertices in $V(G)$ which are adjacent to $u$ and the closed neighborhood of such a vertex $v$, is $N[v]=N(v) \cup\{v\}$. Let $D$ be a subset of $V(G)$ and $v \in D$. Then a private neighborhood of a vertex $v \in V(G)$ with respect to $D$ denoted by $P_{r}[v, D]$ is defined as follows: $P_{r}[v, D]=\{\mathrm{w} \in V(G): N(w) \cap D=\{v\}\}$. We note that a) If $w \in V(G)-D$ and $w$ is adjacent only to $v \in D$, then $w \in P_{r}[v, D]$; b) If $w \in D$ and $w \neq v$, then $w \notin P_{r}[v, D]$; c) If $w=v$ is not adjacent to any vertex

Received: September 25, 2015

(C) 2015 Academic Publications, Ltd. url: www.acadpubl.eu

${ }^{\S}$ Correspondence author 
of $D$, then $w \in P_{r}[v, D]$.

By a support vertex of a graph $G$ we mean a vertex that is adjacent to at least one end vertex of $G$. To clarify a support vertex further, consider the graph $P=v_{1} v_{2} v_{3} v_{4} v_{5} v_{6}$, a path on 6 vertices. By a support vertex for $\mathrm{P}$, we mean the vertices $v_{2} / v_{5}$ as they are the vertices which are adjacent to one degree vertices $v_{1} / v_{6}$ respectively. A set $D$ of vertices in a graph $G=(V, E)$ is a dominating set of $G$ if every vertex in $V \backslash D$ is adjacent to some vertex in $D$. The domination number $\gamma(G)$ of $G$ is the minimum cardinality of a dominating set. A total dominating set $D$ of $G$ is a dominating set such that the induced subgraph $\langle D\rangle$ has no isolates, or if any vertex $v$ of $G$ is adjacent to at least one vertex of the set $D$. The total domination number $\gamma_{t}(G)$ of $G$ is the minimum cardinality of a total dominating set. A dominating set $D$ of $G$ is a global dominating set if $D$ is also a dominating set of $G^{C}$. The global domination number $\gamma_{g}(G)$ of $G$ is the minimum cardinality of a global dominating set. A global dominating set $D$ of $G$ is a total global dominating set, if $D$ is also a total dominating set of $G^{C}$. The total global domination number $\gamma_{t g}(G)$ is the minimum cardinality of a total global dominating set.

A connected dominating set $D$ is said to be a minimal connected dominating set $G$ if and only if for any $v \in D, D \backslash v$ is not a connected dominating set. The concept of $k$-domination is stronger than the concept of domination. There are dominating sets which are not $k$-dominating for $k \geq 2$. Let $G$ be a graph and $k$ be a positive integer. A subset $D$ of $V(G)$ is said to be a $k$-dominating set in $G$ if every vertex $v \in V(G) \backslash D$ is adjacent to at least $k$ vertices of $D$. A $k$-dominating set $D$ in $G$ is said to be minimal $k$-dominating set if for any $v \in D, D \backslash v$ is not a $k$-dominating set. A $k$-dominating set in $G$ with minimum cardinality is called a minimum $k$-dominating set in $G$. The minimum cardinality of a $k$-dominating set, denoted $\gamma_{k}(G)$ is called a $k$-domination number. If $D$ is a $k$-dominating set in $G, \gamma_{k}(G) \leq|D|$. If $k=1$, then $\gamma_{1}(G)=\gamma(G)$. If $D$ is a $k$-dominating set in $G$ then it is also $j$-dominating set for $1 \leq j \leq k$, and $\gamma_{j}(G)=\gamma_{k}(G)$. For a graph $G=(V, E)$, a set $D \subseteq V$ is a strong dominating set if every vertex $v \in V \backslash D$ has a neighbor $u$ in $D$ such that the degree of $u$ is not smaller than the degree of $v$. The minimum cardinality of a strong dominating set of $G$ is the strong domination number, $\gamma_{\text {strong }}(G)$.

We call a vertex $v \in V(G)$, a $P_{G o o d}$-vertex $\left(P_{B a d}\right.$-vertex $)$ with respect to a property $P$, if $v$ belongs to (does not belong to) a $P$-set of either minimum or maximum cardinality. Let $x$ and $y$ denote the number of $P_{\text {Good }}$ and $P_{b a d}$ vertices in a graph $G$. A graph $G$ is called $P_{\text {Excellent }}$ if every vertex in $V$ is $P_{\text {Good }}, P_{\text {Commendable }}$ if $x>y>0, P_{\text {Fair }}$ if $x=y$ and $P_{\text {Poor }}$ if $x<y$ [3]. The $k$-corona of a graph $G$ is the graph obtained from $G$ by identifying an end 
vertex $v_{j}$ of a path of length $k$ with each vertex $v \in V(G)$ and attaching this path to $v$ by letting $v=v_{j}$. For instance, Let $\mathrm{G}=C_{4}=u_{1} u_{2} u_{3} u_{4} u_{1}$. Consider $\mathrm{P}=v_{1} v_{2} v_{3} v_{4} v_{5} v_{6}$, a path of length 6 . Then by the 6-corona of $C_{4}$ we mean the graph in Figure 1. Further by a end vertex of $\mathrm{P}=v_{1} v_{2} v_{3} v_{4} v_{5} v_{6}$ we mean a vertex of degree 1 . Here for $\mathrm{P}$, they are $v_{1}$ and $v_{6}$. We can identify either $v_{1}$ or $v_{6}$ with every vertex of $C_{4}$. In Figure 1 we have identified $v_{1}$ with $u_{1}, u_{2}, u_{3}$ and $u_{4}$. The distance between two vertices $u$ and $v$, denoted $d_{G}(u, v)$ is the length of the shortest path between $u$ and $v$ in $G$. The distance of a vertex $v$ in $G$ is defined as $d_{G}(v)=\Sigma d_{G}(u, v)$. That is the distance of a vertex $v$ in a graph $G$ is the sum of the distances from $v$ individually to all other vertices of $G$. The eccentricity of a vertex $v$ in $G$ denoted by $e(v)$ is the number of $\max _{u \in V(G)} d_{G}(u, v)$. Note that the $\operatorname{diam}(G)$, called the diameter of $G$ is the maximum eccentricity.

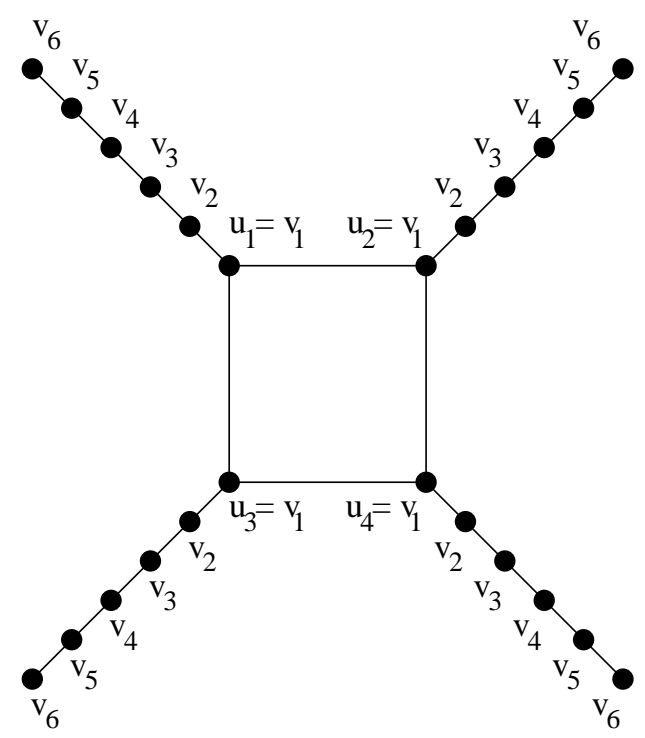

Figure 1: A 6-Corona with $C_{4}$ as base graph 


\section{General Results}

\subsection{Outer Connected Domination Number}

For a given graph $G=(V, E)$, a set $D \subseteq V(G)$ is said to be an outer connected dominating set if $D$ is dominating and the graph induced by $V(G \backslash D)$ is connected. The outer connected domination number of $G$, denoted $\gamma_{c}^{\sim}(G)$ is the cardinality of a minimum outer connected dominating set of $G$ [1]. Note that every graph $G$ has an outer connected dominating set. This is because the set of all vertices of $G$ is an outer connected dominating set in $G$. It is obvious that if $G$ is a graph of order $n$, then $1 \leq \gamma_{c}^{\sim}(G) \leq n$. In addition, $\gamma_{c}^{\sim}(G)=1$ if and only if $G=K_{1} \vee H$, where $H$ is a connected graph of order $n-1$, while $\gamma_{c}^{\sim}(G)=n$ if and only if $G=K_{n}^{c}$. Hence $\gamma_{c}^{\sim}(G) \leq n-1$ if $G$ has at least one edge. Also $\gamma_{c}^{\sim}(G) \neq n-2$ if and only if $G$ has at least one edge which is not an end-edge. In general, $\gamma_{c}^{\sim}(G) \leq n-k$ if and only if there exists a proper connected subgraph $H$ of $G$ such that $|V(H)|=k$ and every vertex of $H$ has a neighbor which belongs to $V(G) \backslash V(H)$. It is easy to see that if $G$ is a connected graph on $n \geq 2$ vertices, then $\gamma_{c}^{\sim}(G)=n-1$ if and only if $G$ is a star. This is because, if $\mathrm{G}$ is a star $K_{1, n-1}$ with $V\left(k_{1, n-1}\right)=\left\{u, u_{1} \ldots u_{n-1}\right\}$ and edge set $E\left(k_{1, n-1}\right)=\left\{u, u_{i}: 1 \leq i \leq n-1\right\}$ then any outer connected dominating set $\mathrm{D}$ of this graph cannot contain $u$. Further it cannot miss any $u_{i}$ for $1 \leq i \leq n-1$. Therefore it follows that $\gamma_{c}^{\sim}(G)=n-1$. Conversely it is equally trivial to see that if $\gamma_{c}^{\sim}(G)=n-1$ for a graph $\mathrm{G}$ then it must be a star.

Theorem 1. An outer connected dominating set $D$ of $G$ is minimal if and only if for each vertex $v \in D$ one of the following holds good: a) $\left.P_{r}[v, D] \neq \emptyset ; b\right)$ $v$ is an isolated vertex in the graph induced by $D ; c) N(v) \cap(V \backslash D)=\emptyset$.

Proof. First we prove the sufficiency. If none of the three conditions hold good under the supposition of the necessity part then there exists a vertex $v \in D$ such that $D^{\prime}=D \backslash\{v\}$ is a dominating set of $G$. But as $N(v) \cap\left(V \backslash D^{\prime}\right) \neq \emptyset$, it follows that the graph induced by $\left(V \backslash D^{\prime}\right)$ is connected. This means that $D^{\prime}$ is a outer connected dominating set of $G$ with lesser number of elements than $D$, a contradiction. Next, it is easy to prove the necessity under the supposition of the sufficiency part.

Theorem 2. For any graph $G, \gamma_{c}^{\sim}(G) \leq n-w(G)+1$ where $w(G)$ is the clique number of $G$. 
Proof. Let $A$ be a set of vertices of $G$ such that the subgraph induced by $A$, denoted $\langle A\rangle$ is complete with $|A|=w(G)$. Now, for any $u \in A$, as $(V \backslash A) \cup\{u\}$ is an outer connected dominating set of $G$ the result follows.

Theorem 3. If $\kappa(G)>\beta_{0}(G)$, then $\gamma_{c}^{\sim}(G)=\gamma(G)$, where $\kappa(G)$ is the vertex connectivity of $G$ and $\beta_{0}(G)$ is the vertex independence number of $G$.

Proof. Let $D$ be a dominating set of $G$. Since $\gamma(G) \leq \beta_{0}(G) \leq \kappa(G)$, it follows that $V \backslash D$ is connected. But then as $\kappa(G) \leq \gamma_{c}^{\sim}(G)$ the result follows.

Theorem 4. Let $D$ be a outer connected dominating set of a connected graph $G$. If no two vertices in $V \backslash D$ are adjacent to a common vertex of $D$ then $\gamma_{c}^{\sim}(G)+r(T) \geq n$ where $r(T)$ is the maximum number of pendant vertices of any spanning tree $T$ of $G$.

Proof. By the hypothesis of the theorem, we have for any vertices $u, v \in$ $V \backslash D$, there exist two vertices $u_{1}, v_{1} \in D$ such that $\left(u_{1}, u\right) \in E(G)$ and $\left(v_{1}, v\right) \in$ $E(G)$ but $\left(u_{1}, v\right),\left(v_{1}, u\right) \notin E(G)$. Therefore there exists a spanning tree $T$ of $V \backslash D$ in which each vertex of $V \backslash D$ is adjacent to a vertex of $D$. This shows that $r(T) \geq|V \backslash D|$. Hence it follows that $\gamma_{c}^{\sim}(G)+r(T) \geq n$.

\subsection{Doubly Connected Domination Number}

We say that a set $D \subseteq V(G)$ is a doubly connected dominating set of $G$ if it is dominating and the induced subgraphs $G[D]$ and $G[V(G) \backslash D]$ are connected. The cardinality of a minimum doubly connected dominating set in $G$ is called a doubly connected domination number and is denoted by $\gamma_{c c}(G)$ [2]. Since for an arbitrary graph $G$, every connected dominating set is a dominating set and every doubly connected dominating set is a connected dominating set, we have $\gamma(G) \leq \gamma_{c}^{\sim}(G) \leq \gamma_{c c}(G)$.

Theorem 5. A doubly connected dominating set $D$ of $G$ is minimal if and only if for each vertex $v \in D$ one of the following conditions hold good: a) $\left.\left.P_{r}[v, D] \neq \emptyset ; b\right) N(v) \cap(V \backslash D)=\emptyset ; c\right) v$ is a cut vertex of the subgraph induced by $D$.

Proof. First assume the necessity and let $D$ be a doubly connected dominating set. The definition of doubly connectedness implies that the subgraphs induced by $D$ and $V \backslash D$ are both connected. We know from Theorem 9.1 of [6] that a connected dominating set $D$ satisfies either of $a$ ) or $c$ ) given in the 
statement. Moreover as $\langle V \backslash D\rangle$ is connected, it also satisfies the condition $b$ ). Further it is easy to see that the converse is also true under the supposition of the sufficiency part. One can also refer to $[4,5]$.

\subsection{2-Packing Number}

Define a 2-packing of a graph $G$ as a set $X \subset V(G)$ of vertices such that $N[x] \cap N[y]=\emptyset$ for each pair of distinct vertices $x, y \in X$. Alternatively, we can define a 2-packing of $G$ as a set $X$ of vertices in $G$ such that for any pair of vertices $x$ and $y$ in $X, d(x, y)>2$. The maximum cardinality of a 2-packing set of $G$ is called the 2-packing number of $G$ and is denoted by $\rho_{2}(G)$.

Theorem 6. For any graph $G, \rho_{2}(G) \leq \gamma(G)$.

Proof. Let $S$ be a maximal 2-packing set of $G$. Then as $d(u, v)>2$ for every pair of vertices $u$ and $v$ in $S$, we need at least one vertex in $V(G)$ to dominate each vertex in $S$. Hence, the cardinality of a minimal dominating set is greater than or equal to the the cardinality of a maximal 2-packing set.

Theorem 7. Every graph $H$ is an induced subgraph of a $\gamma_{c^{-}}$Commendable graph.

Proof. Let $H$ be a graph. Let $G$ be a 3-corona of $H$ such that for each $u_{i} \in V(H)$, we add vertex $u_{i}^{\prime}$ and edge $u_{i} u_{i}^{\prime}$ to the 3-corona of $H$. Clearly $H$ is an induced subgraph of $G$. We now claim that $G$ is $\gamma_{c}$-Commendable. Let $\left\{H_{i}\right\}, 1 \leq i \leq k$ be the components of $H$. Two cases arise.

Case 1: $\left|V\left(H_{i}\right)\right|=1$

Here each $H_{i} \cong K_{1}$, say the vertex $v_{i}$. In $G, v_{i}$ is a support vertex of a $P_{5}$. From Table.1 we know that every path $P_{n}$ for $n \geq 5$ is $\gamma_{c}$-Commendable. Then $P_{5}$ has a unique $\gamma_{c}$-set and $\gamma_{c}\left(P_{5}\right)=3$. So for every $K_{1}$ in $H$, we have a $\gamma_{c}$-Commendable subgraph in $G$.

Case 2: $\left|V\left(H_{i}\right)\right|>1$

Here each vertex $u_{j} \in V\left(H_{j}\right)$ is a support vertex in $G$. Further, each $u_{j}$ is adjacent to at least one other support vertex $u_{l} \in G$. Notice that these support vertices dominate each other and their corresponding end vertices $u_{j}^{\prime}$ and $u_{l}^{\prime}$ cannot be in any $\gamma_{c}$-set of $G$. Moreover these are the only bad vertices in $G$ with respect to $\mathrm{CD}$ property. As each subgraph of $G$ is $\gamma_{c}$-Commendable it follows that $x>y$ for the entire graph $G$.

Theorem 8. Every graph $H$ is an induced subgraph of a $\gamma_{c}$-Excellent graph. 
Proof. Every graph $H$ is an induced subgraph of the 2-corona of $H$. Let $G$ be a 2-corona of a graph $H$. Observe that every $\gamma_{c}$-set of $G$ must contain all the support vertices of $G$ along with a neighbor for each such support vertex. That is, if $u$ is a support vertex in $G$, then $u$ is in every $\gamma_{c}$-set of $G$ and at least one neighbor of $u$ is in every $\gamma_{c}$-set. So if $T_{1}$ is the set of all support vertices in $G$ and $T_{2}$ is the set of all end vertices in $G$, then $T_{1} \cup T_{2}$ and $T_{1} \cup V(H)$ are both $\gamma_{c}$-sets of $G$. Therefore $G$ is $\gamma_{c}$-Excellent.

Theorem 9. The 1-corona of any connected graph $H$ is $\gamma_{c}$-Fair.

Proof. Let $G=H \circ K_{1}$ be a 1-corona of $H$. Then it is easy to see that $|V(G)|=2|V(H)|$. So the order of $G$ is even. Note that we require all vertices of $H$ to dominate their end vertices and hence $\gamma_{c}(G)=|V(H)|$. Moreover we observe that the connected dominating set $V(H)$ is the set of all support vertices of $G$. This property precludes the possibility of the inclusion of any end vertex. Therefore $G$ is $\gamma_{c}$-Fair.

Corollary 10. Every connected graph is an induced subgraph of a $\gamma_{c}$-Fair graph.

Theorem 11. Every connected graph $H$ is an induced subgraph of a $\gamma_{c}$-Poor graph.

Proof. Construct a new graph $G$ from a given connected graph $H$ by identifying the central vertex of a star $K_{1, r}$ with every vertex of $H$. Then clearly $H$ is an induced subgraph of $G$. As $H$ is connected, every vertex $u \in V(H)$ has a neighbor in $V(H)$. The very construction of $G$ reveals that every vertex of $H$ in $G$ is a support vertex. Moreover every support vertex in $G$ is adjacent to some other support vertex in $G$, one dominating the other along with their adjacent end vertices. So $G$ has a unique $\gamma_{c}$-set, $V(H)$ with the property that each vertex $u \in V(H)$ has $r$ end vertices adjacent to it, of which none will be in the $\gamma_{c}$-set. That is, $G$ has, for $|V(H)|$ good vertices, $r|V(H)|$ bad vertices. Hence $G$ is $\gamma_{c}$-Poor.

Theorem 12. For every positive integer $r \geq 1$, there exists a graph $G$ such that $\gamma_{c c}\left(G \backslash\left\{v_{1}, v_{2}\right\}\right)-\gamma_{c c}(G)=r$.

Proof. Consider the cycle $C_{r+4}, r \geq 1$. Obtain a new graph $G$ from $C_{r+4}$ by joining each vertex of $C_{r+4}$ with every vertex of a $K_{2}$ graph. That is $G=$ $C_{r+4} \vee K_{2}$. Obviously the vertices of $K_{2}$ are global non-cut vertices of $G$ and $\gamma_{c c}(G)=2$. If we remove the vertices $v_{1}, v_{2}$ of $K_{2}$ from $G$ then $G \backslash\left\{v_{1}, v_{2}\right\} \cong$ 
$C_{r+4}$. We know that $\gamma_{c c}\left(C_{r+4}\right)=r+2$. So from Table.1 $\gamma_{c c}\left(G \backslash\left\{v_{1}, v_{2}\right\}\right)-$ $\gamma_{c c}(G)=(r+2)-2=r$.

Theorem 13. For every connected graph $G$ and a vertex $v \in V(G)$ such that $G \backslash v$ is connected we have $\gamma_{c c}(G) \leq \gamma_{c c}(G \backslash v)+1$.

Proof. Let $D$ be a minimum connected dominating set of $G \backslash v$. Then $D \cup\{v\}$ will be a minimum doubly connected dominating set of $G$ and hence $\gamma_{c c}(G) \leq \gamma_{c c}(G \backslash v)+1$.

Theorem 14. For any graph $G, \underset{c}{\sim} \leq|V(G)|-\operatorname{diam}(G)+s+1$ where $s$ is the minimum number of vertices in a $\gamma_{c}^{\sim}$-set $D$ of $G$ that lie in the shortest diametrical $x-y$ path.

Proof. Suppose that $\operatorname{diam}(G)=t$. Then three possibilities arise. First, it may happen that $x, y \in V \backslash D$. Then note that in such an instance, $V \backslash D$ has at least $t+1$ vertices. Second it may be the case that $x \in D$ and $y \in V \backslash D$. If there exists a vertex $x_{1} \in V \backslash D$ such that $x_{1}$ is connected to $x$ through the vertices of $D$. Then it follows that $d(x, y) \geq k-(t+1)$ and hence $V \backslash D$ has at least $t-s$ vertices. This is so, because, in the event of the opposite, there exists a vertex $z$ adjacent to every $x_{1} \in V \backslash D$ such that $d(x, z)=d(x, y)+d\left(y, x_{1}\right)+d\left(x_{1}, z\right) \geq$ $t+1$, a contradiction. So in this case, we have $V \backslash D=\{y\}$ and $G \cong K_{2}$, or $K_{1,2}$. Finally, it may be the instance that $x, y \in D$. Suppose that there exists $x_{1}, y_{1} \in V \backslash D$ such that $x$ is connected to $x_{1}$ and $y$ is connected to $y_{1}$ through the vertices of $D$. Then $d\left(x_{1}, y_{1}\right)$ is at least $t-(s+2)$ and hence $V \backslash D$ has at least $(t-s-1)$ vertices. This is because, otherwise there will exist exactly one vertex $x_{1} \in V \backslash D$ that is adjacent to both $x$ and $y$ and $V \backslash D=\left\{x_{1}\right\}$ and as a consequence it has $G \cong K_{1,3}$. So in all the three instances, $V \backslash D$ has at least $(t-s-1)$ vertices. 


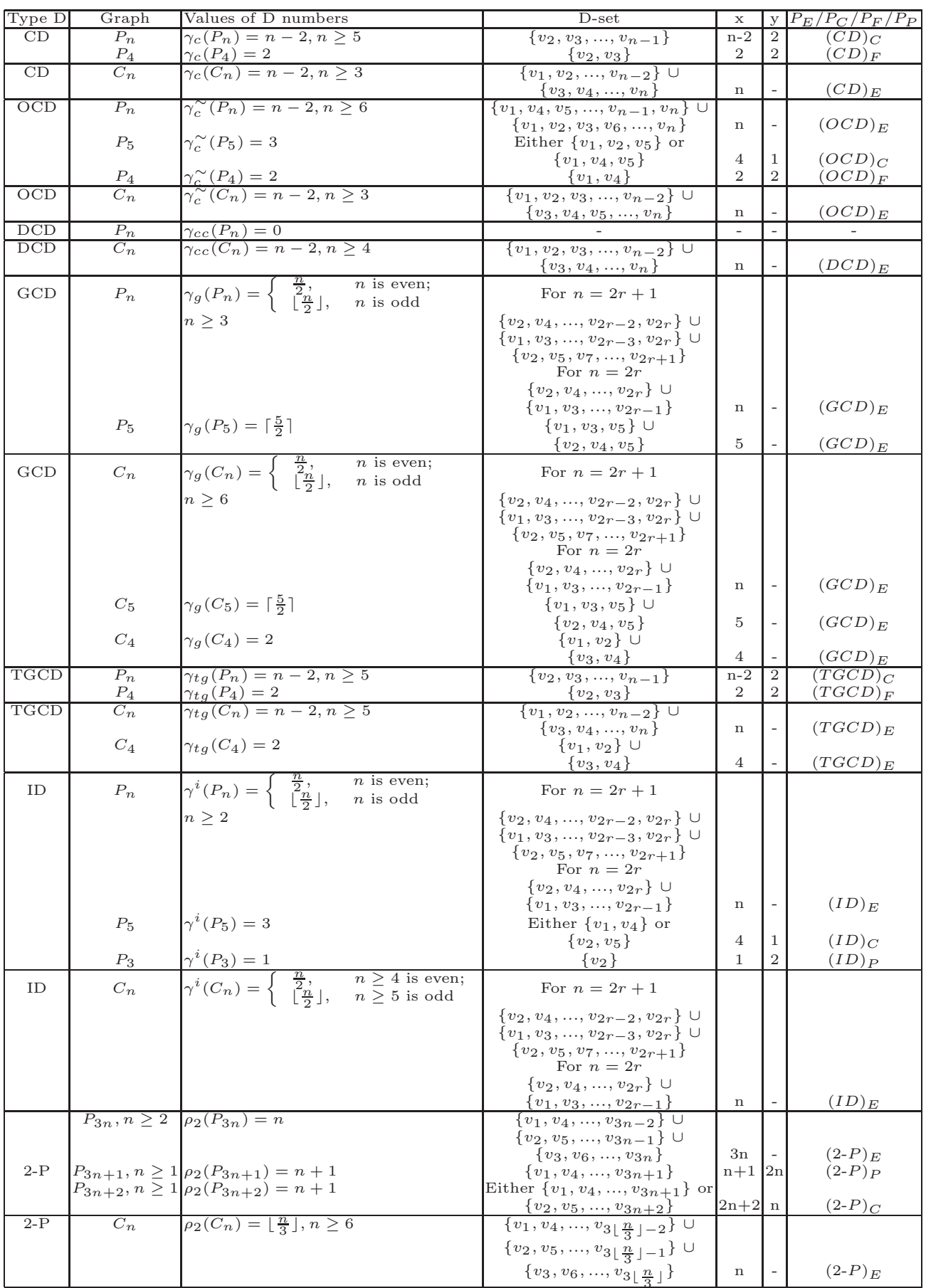

Table 1: A summary of Values of Various Domination Numbers of $P_{n}$ and $C_{n}$ 


\section{Values of Various Domination Numbers of $P_{n}$ and $C_{n}$}

We need to compute the domination numbers such as Connected Domination (CD), Outer Connected Domination (OCD), Doubly Connected Domination (DCD), Global Connected Domination (GCD), Total Global Connected Domination (TGCD), Independence Domination (ID), 2-Packing (2-P) for $P_{n}=$ $v_{1} v_{2} v_{3} \ldots v_{n-1} v_{n}$ and $C_{n}=v_{1} v_{2} v_{3} \ldots v_{n-1} v_{n} v_{1}$. As the process of computation of these parameters are mostly similar we have carefully decided to not give the proof of these results individually. Instead we have exhibited the results appropriately in the form of a table. Table 1 summarizes our results concerning the Paths and Cycles.

In Table 1 by a D-set in column 4 we mean the respective dominating set. For instance, the dominating D-set in column 4 and row 1 is a connected dominating set (represented as CD in column 1 and row 1) for the graph $P_{n}$. The respective $\mathrm{D}$-set is: $\mathrm{D}=\left\{v_{2}, v_{3}, \ldots, v_{n-1}\right\}$. Similarly for the graph $P_{4}$, the $\mathrm{D}$-set is: $\mathrm{D}=\left\{v_{2}, v_{3}\right\}$. In both the cases all compulsory elements are included as per the requirement of the respective definition.

No elements have been lost. The same is true for all types of dominating numbers listed in the Table.1

\section{Abbreviation}

$\mathrm{D} \rightarrow$ Domination $\quad \mathrm{D}$-set $\rightarrow$ Dominating Set $\quad x \rightarrow x$-value $y \rightarrow y$-value $P_{E} \rightarrow P_{\text {Excellent }} \quad P_{C} \rightarrow P_{\text {Commendable }} \quad P_{F} \rightarrow P_{\text {Fair }} \quad P_{P} \rightarrow P_{\text {Poor }}$

\section{References}

[1] Joanna Cyman, The Outer-Connected domination number of a graph, $A u$ tralasian Journal of Combinatorics, 38 (2007), 35-46.

[2] H. Karami, R. Khoeilar and S.M. Sheikholeslami, Doubly Connected Domination Subdivision Numbers of Graphs, MATEMA, 64, No. 3 (2012), 232239.

[3] Robert Elmer Dautermann III, Vertices in Total Dominating Sets, Electronic Theses and Dissertations, East Tennessee State University, May 2000 .

[4] V. Yegnanarayanan and G.K. Umamaheswari, On Rolf Nevanlinna Prize Winners Collaboration Graph, JCMCC, 79 (2011), 43-58. 
[5] V. Yegnanarayanan and G.K. Umamaheswari, On Rolf Nevanlinna Prize Winners Collaboration Graph-II, International Journal of Scientific and Research Publications, 2(4) (2012), 354-367.

[6] V. Yegnanarayanan and G.K. Umamaheswari, On Rolf Nevanlinna Prize Winners Collaboration Graph-III, Journal of Mathematical and Computational Sciences, 3(2) (2013), 419-455. 
